\title{
Diversidad de mamíferos medianos en el Parque Nacional Pico de Orizaba
}

Diversity of medium mammals in the Pico de Orizaba National Park

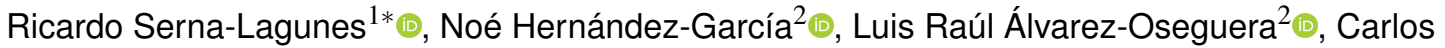

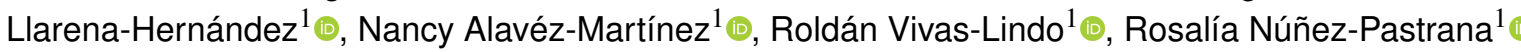 \\ ${ }^{1}$ Facultad de Ciencias Biológicas y Agropecuarias, región Orizaba-Córdoba, Universidad Veracruzana. Josefa Ortiz de Domínguez, s/n, \\ Col. Centro, Peñuela. CP. 94945. Amatlán de Los Reyes, Veracruz, México. \\ ${ }^{2}$ Comisión Nacional de Áreas Naturales Protegidas. Poniente 20 28, Col. Sta Maria Tlachichilco. CP. 94350. Orizaba, Veracruz, México. \\ *Autor de correspondencia: rserna@uv.mx
}

Artículo científico recibido: 25 de noviembre de 2018 aceptado: 30 de julio de 2019

RESUMEN. El Parque Nacional Pico de Orizaba (PNPO) es la montaña más elevada del territorio mexicano que protege ecosistemas templados. Tala clandestina, incendios forestales, cambio de uso de suelo e incremento en la afluencia turística, afectan la estructura de la comunidad de mamíferos y su estudio permitirá el diseño de estrategias oportunas de conservación. El objetivo fue analizar la riqueza de mamíferos del PNPO en la época de secas y lluvias, evaluar su estado de conservación y clasificarlos por gremios tróficos. De enero a diciembre del 2017, se activaron 14 cámaras-trampa (distancia promedio entre cámara de 0.5-2 km) para el registro de mamíferos. Las fotocapturas se clasificaron en registros independientes y se calcularon indicadores como cobertura del muestreo $(\breve{C})$, riqueza, abundancia, gremios tróficos y categoría de riesgo. La $\check{C}$ n fue superior al $95 \%$ en cada época, indicador de un muestreo representativo al registrar 10 especies $\left({ }^{q} \mathrm{D}\right.$ = 9 especies en época de lluvias; ${ }^{q} \mathrm{D}=8$ especies en época de secas) de ocho géneros y pertenecientes a seis familias. Los mamíferos presentaron distinta riqueza y abundancia entre épocas, pero no fue significativamente diferente cuando se comparó el índice de Shannon entre épocas ( $p>0.05$ ). El orden Carnívora fue el mejor representado con el $60 \%$ de mamíferos, resultado de una red trófica desbalanceada. Leopardus wiedii, Conepatus semistriatus y Sciurus oculatus se encuentran en riesgo, su conservación puede ser mediante prácticas de mejoramiento de su hábitat y la gestión del PNPO puede considerarlas bajo el concepto de especies bandera.

Palabras clave: Área natural protegida, mamíferos en riesgo, mastofauna, mejoramiento de hábitat.

ABSTRACT. The Pico de Orizaba National Park (PNPO) is the highest mountain in the Mexican territory that protects temperate ecosystems. Clandestine logging, forest fires, change in land use and increased tourist influx affect the structure of the mammalian community and its study will allow the design of appropriate conservation strategies. The objective was to analyze the richness of mammals of the PNPO in dry and rainy season, evaluate their conservation status and classify them by trophic guilds. From January to December 2017, 14 trap cameras (average distance between 0.5-2 km camera) were activated for mammalian registration. The photocaptures were classified into independent records and indicators such as sampling coverage $(\check{C} n)$, richness, abundance, trophic guilds and risk category were calculated. The $\check{C}$ n was greater than $95 \%$ at each season, an indicator of a representative sample when registering 10 species $\left({ }^{q} \mathrm{D}=9\right.$ species in the rainy season; ${ }^{q} \mathrm{D}=8$ species in the dry season) of eight genera and belonging to six families. Mammals presented different richness and abundance between periods, but it was not significantly different when the Shannon index was compared between periods ( $p$ $>0.05$ ). The Carnivorous order was the best represented with $60 \%$ of mammals, resulting from an unbalanced trophic network. Leopardus wiedii, Conepatus semistriatus and Sciurus oculatus are at risk, their conservation can be through habitat improvement practices and the management of the PNPO can consider them under the concept of umbrella species.

Key words: Habitat improvement, mammals at risk, mastofauna, protected natural area. 


\section{INTRODUCCIÓN}

La riqueza de especies de mamíferos ha sido una medida del éxito de conservación de las Áreas Naturales Protegidas en México (ANP, Ceballos 2007). Los estudios de diversidad de mamíferos como un grupo de interés, ha cobrado importancia en los últimos años, siendo uno de los grupos de vertebrados con prioridades de conservación en ANP (Ceballos et al. 1998, Peterson et al. 2000). Las ANP están sujetas a la Ley General del Equilibrio Ecológico y Protección al Ambiente, las cuales se definen cómo zonas del territorio mexicano con ambientes naturales representativos de una región, con bajo impacto por actividades antropogénicas (LGEEPA 2007). El objetivo de una ANP es conservar ecosistemas, su biodiversidad, especies en riesgo como las listadas en la NOM-059-SEMARNAT-2010 (SEMARNAT 2010) y especies endémicas (García-Marmolejo et al. 2008). En este sentido, evaluar la riqueza de mamíferos puede considerarse como un indicador del estado de salud del ecosistema resguardado por el ANP (Vázquez y Valenzuela-Galván 2009). En Veracruz, las ANP están presentes desde 1923, con el decreto de la Reserva Forestal El Gavilán, en el municipio de Minatitlán (Vázquez-Torres et al. 2010). Posteriormente, en la década de los treinta, se decretó el Parque Nacional Cofre de Perote y Parque Nacional Pico de Orizaba (PNPO, DOF 1937). Este último, ubicado entre los límites de los estados de Puebla y Veracruz, con una altura máxima de 5 636 msnm, es la montaña más elevada del territorio mexicano y por ende brinda protección a ecosistemas de alta montaña de México (CONANP 2015).

Acerca de la fauna que habita el PNPO se ha estimado una riqueza de vertebrados que asciende a 159 especies (47 mamíferos, 48 anfibios y reptiles y 64 aves), de los cuales, 30 son endémicos y 44 especies están protegidas por la legislación mexicana (CONANP 2015). De las especies de mamíferos registradas para el ANP, 10 están en la NOM059-SEMARNAT-2010 (SEMARNAT 2010), entre los que destacan: ardilla de Peter (Sciurus oculatus), musaraña dientuda (Sorex macrodon), musaraña oscura (Sorex oculatus), musaraña de Saussure (Sorex veraecrucis), ardilla voladora del sur (Glaucomys volans), musaraña orejullas de Goldman (Cryptotis alticola), murciélago trompudo (Choeronycteris mexicana), murciélago con cola (Enchisthenes hartii), murciélago hocicudo mayor (Leptonycteris nivalis) y ratón de Nelson (Megadotomys nelsoni) (Fa y Morales 1991, Ceballos y Oliva 2005, Gámez et al. 2012). Otras especies como lince (Lynx rufus), coyote (Canis latrans) y sus presas potenciales, han sido objeto de estudio sobre sus patrones actividad en el PNOP, indicando las prioridades de conservación de este grupo (Serna-Lagunes et al. 2019). Sin embargo, los incendios forestales, la tala clandestina, el cambio de uso de suelo para la ganadería, agricultura, asentamientos humanos y el incremento en la afluencia turística (Cerano-Paredes et al. 2016, Mejía-Ronzón et al. 2016), impacta de manera negativa a la estructura y dinámica del ensamblaje de mamíferos, el cual debe ser evaluado oportunamente para diseñar estrategias efectivas de conservación (Peterson et al. 2000).

Los mamíferos han sido considerados como grupo indicador de diversas condiciones de los ecosistemas, como de la alteración del hábitat, debido a que ciertas especies son indicadoras de la salud y calidad del ecosistema (Rumiz 2010). Existen dos maneras de evaluar los cambios en la estructura de la comunidad de mamíferos: la riqueza de especies y la diversidad de gremios tróficos (Magurran 1988, Simberloff y Dayan 1991, Morin 2011). Si se estudian ambas características, se describe la estructura de la comunidad y se infiere sobre la forma de cómo los mamíferos hacen uso de los recursos (PérezIrineo y Santos-Moreno 2013). Por lo anterior, este estudio tuvo como objetivo analizar la riqueza de mamíferos de talla mediana en la época de secas y de lluvias en el PNPO, además de evaluar su estado de conservación e identificar los gremios tróficos de los mamíferos registrados.

\section{MATERIALES Y MÉTODOS}

\section{Área de Estudio}

El estudio se realizó en el Parque Nacional Pico de Orizaba ( $18^{\circ} 56^{\prime} 30^{\prime \prime}$ y $19^{\circ} 09^{\prime} 30^{\prime \prime} \mathrm{LN}, 97^{\circ}$ 
12 ' $30^{\prime \prime}$ y $97^{\circ} 22$ ' $30^{\prime \prime}$ LO), el cual comprende un área total de 19756 ha, con una fracción del polígono en el Estado de Puebla (Tlachichuca, Chalchicomula de Sesma, Atzitzintla) y Veracruz (Chalcahualco y La Perla) (Figura 1). El intervalo altitudinal va de 2 700 a $5760 \mathrm{msnm}$, en el borde de la Meseta Central (Vargas 1984). Existen tres tipos de vegetación dentro del PNPO: bosque de pino (Pinus patula, $P$. pseudostrobus y $P$. montezumae) que se encuentra de 2000 a $3000 \mathrm{~m}$, donde el estrato arbóreo alcanza hasta $25 \mathrm{~m}$ de altura; vegetación subalpina de 3000 a $4000 \mathrm{~m}$ y páramo de altura de 4000 a 4 200 m (SARH 1993, Martínez-Vázquez et al. 2010, CONANP 2015).

\section{Diseño del muestreo y duración del monitoreo}

El estudio se realizó de enero a diciembre de 2017. Se utilizó el método de captura indirecta con la técnica de fototrampeo para el registro de mamíferos de acuerdo con la propuesta de instalación de las fototrampas de Chávez et al. (2013). Se colocaron 14 cámaras-trampas dentro del PNPO, cuyos modelos utilizados fueron Cuddeback 1231 infrarrojo Black Flash $E 3^{\circledR}$ y Ltl Acorn ${ }^{\circledR}$. Las cámaras-trampa se colocaron de manera permanente sin atrayentes, ya que estos se usan para evaluar la abundancia de carnívoros, aunque sesga el índice de actividad (Harrison 1997) y afectan la incidencia y comportamiento de otros mamíferos, por lo que se recomienda no utilizarlos para estudios de diversidad (Pacheco et al. 2003). Las cámaras se sujetaron a fustes de árboles a una altura no mayor de 50 $\mathrm{cm}$ del suelo y se ubicaron a orillas de cuerpos de agua, sobre senderos donde previamente se identificaron rastros de mamíferos, comederos y bordes de los cerros. Cada trampa permaneció activa las $24 \mathrm{~h}$ del día, con un intervalo mínimo de retraso entre fotografías de 0.3 y $1 \mathrm{~min}$, y en el caso de Cuddeback se tomó un vídeo de 15 seg; las cámaras se colocaron a una distancia promedio entre $500 \mathrm{~m}$ y $2 \mathrm{~km}$ entre cámaras, para evitar sesgo (conteos dobles) de las especies registradas (Díaz-Pulido y Payán 2012). Seis cámaras-trampa se ubicaron en la vertiente Oeste y el resto en la vertiente Este, con la intención de rodear el cráter del Volcán. También, se georreferenció cada estación de muestreo (Figura 1).

\section{Análisis de datos}

En el análisis se utilizaron registros (eventos) independientes de cada especie, considerándose como tal a individuos: 1) en fotografías consecutivas de ejemplares de la misma especie plenamente identificables, 2) en fotografías con una separación de $3 \mathrm{~h}$ en las que no fue posible identificar a cada ejemplar de la misma especie y 3) a cada individuo en fotografías de múltiples individuos (ÁvilaNájera et al. 2016). La identificación taxonómica de los mamíferos fotocapturados se realizó con literatura especializada (Aranda 2012). La nomenclatura de las especies se revisó en Wilson y Reeder (2005, 2011) y se actualizaron con base en Ramírez-Pulido et al. (2014). Los mamíferos registrados se clasificaron de acuerdo con su tipo de alimentación en los ensambles: carnívoros, herbívoros, insectívoros y omnívoros (Ceballos y Navarro 1991, Pérez-Irineo y Santos-Moreno 2013). Por último, el estatus de conservación se constató en la NOM-059-SEMARNAT2010 (SEMARNAT 2010).

El esfuerzo de colecta se expresó como el número de trampas colocadas multiplicado por el total de días que estuvieron activas (trampas*día, PérezIrineo y Santos-Moreno 2013). La completitud del inventario de especies se calculó con el estimador de cobertura de la muestra $(\breve{C} n)$ que indica la proporción de la comunidad total representada por las especies recolectadas y permite comparar la diversidad entre los ensamblajes muestreados a la misma completitud del muestreo; cuando $\breve{C} \mathrm{n} \approx 100 \%$ el muestreo está completo dado el esfuerzo y la técnica de recolección utilizada (Chao y Jost 2012). Lo anterior, debido a que la comparación de la riqueza de especies es apropiada para ensambles con un nivel similar de completitud del inventario (Magurran 2008). Se estimó la riqueza de especies para cada época de muestreo utilizando inter y extrapolaciones basadas en el estimador $\check{C}$ n en el paquete iNEXT (Chao et al. 2016). Se consideró el 95\% como nivel confiable de $\check{C}$ n para comparar la riqueza entre épocas de muestreo utilizando los intervalos de confianza al $95 \%$ en los que las diferencias significati- 

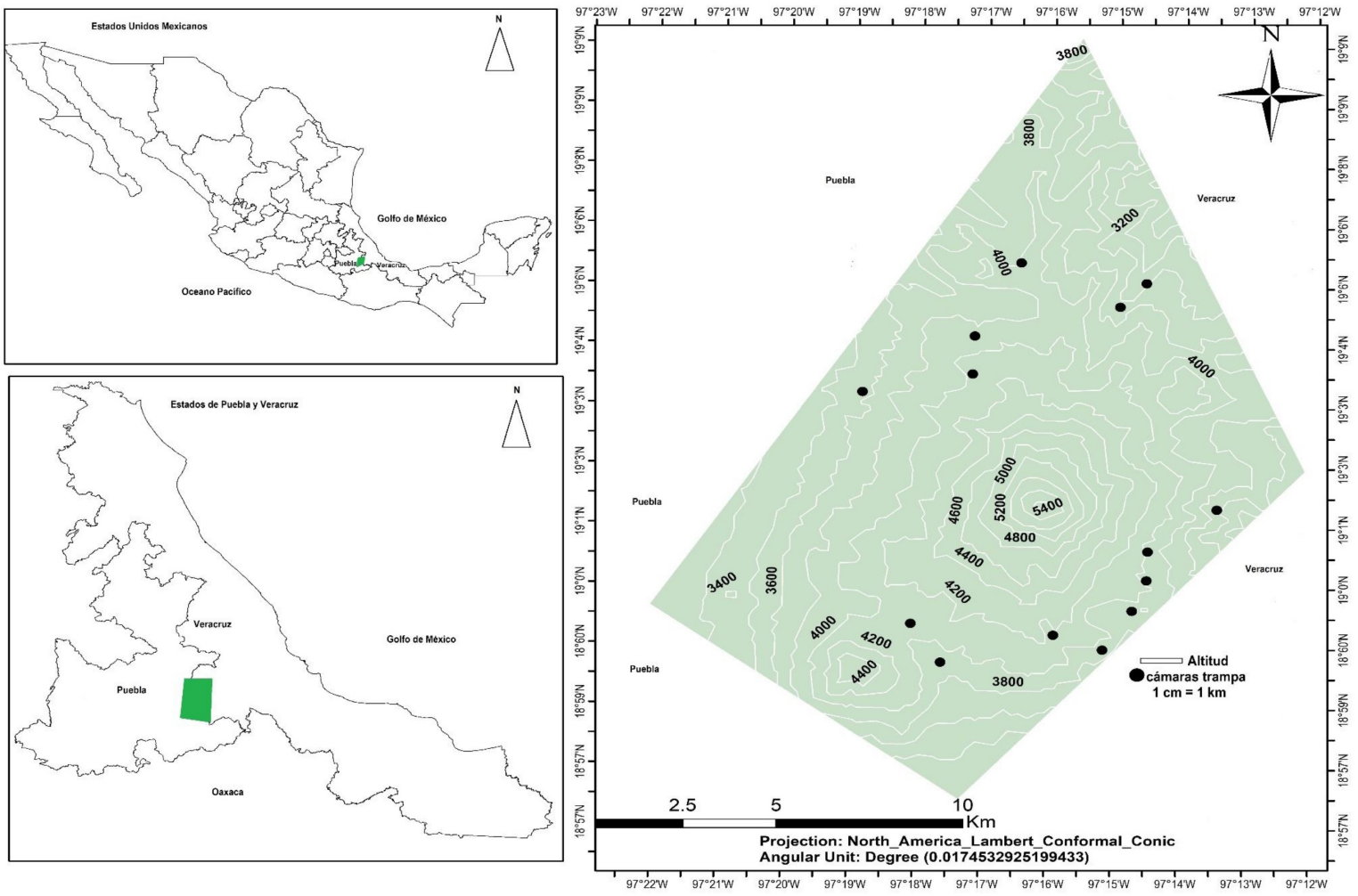

Figura 1. Polígono del PNPO y disposición de las cámaras trampa para el monitorio de mamíferos medianos.

vas se indican mediante la ausencia de superposición entre estos (Cumming et al. 2007, Gotelli y Colwell 2011, Chao y Jost 2012).

De acuerdo con Magurran (1988) se estimaron los indicadores básicos de la diversidad alfa como la riqueza específica y abundancia $(\mathrm{N})$ por época de muestreo, riqueza total (diversidad gama) y diversidad beta $(\beta)$ de especies compartidas. Para describir la estructura de la comunidad de mamíferos en el PNPO, la riqueza específica $\left({ }^{q} D\right)$ en la época de secas y lluvias se calculó cómo el recíproco de un promedio de las abundancias relativas (Hill 1973) con el software TrueDiversity (Goepel 2012). La diversidad compartida (diversidad $\beta$ ) se estimó a partir de curvas de rango-abundancia mediante una salida gráfica que permite comparar la abundancia absoluta de mamíferos registrados entre las épocas (lluvias y secas) de muestreo (Chávez et al. 2013). Se calculó el índice de Shannon-Winner ( $H^{\prime}$ ) para la temporada de secas y para la temporada de lluvias; estos valores se compararon con una prueba de t a un $\alpha=0.05$ con el software Biodiversity Calculator (Danoff-Burg y Chen 2005), para detectar cambios en la estructura de la comunidad de mamíferos entre épocas.

\section{RESULTADOS}

Con un esfuerzo de muestreo de 4928 díastrampas, se obtuvieron 191 registros independientes, y una diversidad gama de ${ }^{q} \mathrm{D}=10$ taxones para el muestreo global, los cuales incluyen seis especies del orden Carnívora, dos del orden Rodentia; Lagomorpha y Cingulata con una especie cada uno. Dos de las 10 especies están en la categoría de Protegida (Pr) y una en Peligro (P) (Tabla 1). Con el perfil de diversidad obtenido con el paquete iNext, los resultados indican que para la época de secas se registraron 8 especies y 67 individuos con un $\breve{C} \mathrm{n}=98.5 \%$, mientras que en la época de lluvias fueron 9 especies y 124 individuos con $\check{C} \mathrm{n}=98.4 \%$. Por lo que en ambos 
Tabla 1. Listado sistemático, taxonómico y registros independientes (RI) en la época de secas y lluvias de la riqueza de mamíferos del Parque Nacional Pico de Orizaba.

\begin{tabular}{|c|c|c|c|c|c|c|}
\hline Orden & Familia & $\begin{array}{l}\text { Nombre } \\
\text { científico }\end{array}$ & $\begin{array}{l}\text { Nombre } \\
\text { común }\end{array}$ & $\begin{array}{c}\text { NOM } \\
059\end{array}$ & $\begin{array}{c}\mathrm{Rl} \text { en la } \\
\text { época de secas }\end{array}$ & $\begin{array}{c}\text { RI en la } \\
\text { época de lluvias }\end{array}$ \\
\hline \multirow[t]{6}{*}{ Carnivora } & Felidae & Lynx rufus & Lince & & 22 & 23 \\
\hline & Felidae & Leopardus wiedii & Tigrillo & $\mathrm{P}$ & 0 & 1 \\
\hline & Canidae & Canis latrans & Coyote & & 5 & 22 \\
\hline & Mephitidae & Mephitis macroura & Zorrillo rayado & & 8 & 2 \\
\hline & Mephitidae & Conepatus semistriatus & Zorrillo de espalda blanca sureño & PR & 2 & 1 \\
\hline & Mephitidae & Conepatus leuconotus & Zorrillo de espalda blanca norteño & & 1 & 0 \\
\hline Cingulata & Dasypodidae & Dasypus novemcinctus & Armadillo & & 0 & 3 \\
\hline \multirow[t]{2}{*}{ Rodentia } & Sciuridae & Sciurus aureogaster & Ardilla roja & & 10 & 14 \\
\hline & Sciuridae & Sciurus oculatus & Ardilla de Peter & PR & 14 & 26 \\
\hline Lagomorpha & Leporidae & Sylvilagus floridanus & Conejo castellano & & 5 & 32 \\
\hline
\end{tabular}

casos, la cobertura de la muestra superó el 95\% del completitud (Figura 2). Entre épocas de muestreo no se observaron diferencias significativas en la riqueza de especies, debido a que los intervalos de confianza se sobreponen (Figura 3).

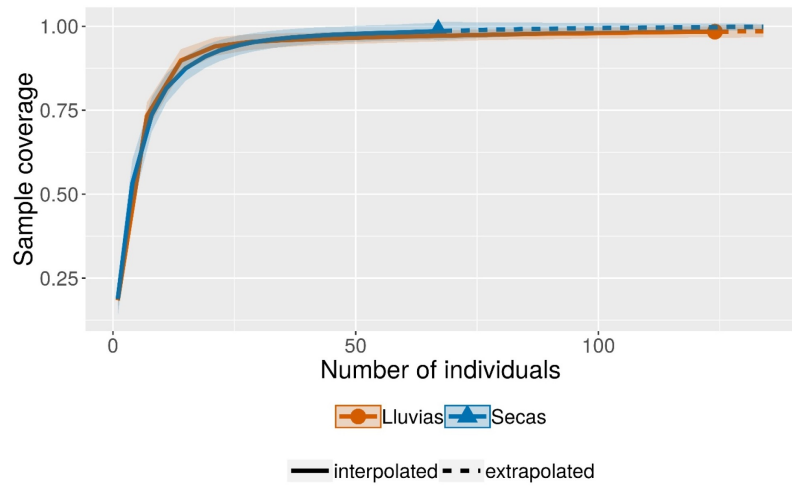

Figura 2. Cobertura de la muestra basada en interpolaciones y extrapolaciones. Tanto en la época de lluvias y secas se obtuvo una completitud superior al 95\% (líneas sombreadas en naranja y azul, respectivamente)

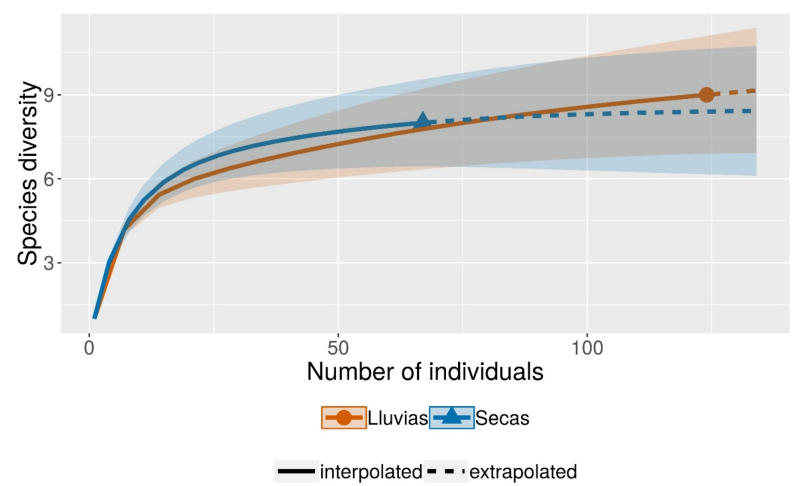

Figura 3. Curva de acumulación de especies que determina la completitud del muestreo en ambas épocas del año. Las curvas sombreadas (naranja y azul) representan los intervalos de confianza remuestreados con 100 réplicas bootstrap.
De los 10 mamíferos registrados, el gremio trófico mayor representado fueron los insectívoros (4 especies); mientras que los omnívoros, herbívoros y carnívoros tuvieron dos especies cada uno (Figura 4). Los mamíferos presentaron distintas abundancias entre épocas (Figura 5). La especie Leopardus weidii fue el mamífero que se presentó sólo en la época de lluvias; mientras que $C$. latrans y $L$. rufus estuvieron presentes en ambas épocas de muestreo, pero en la época de secas $L$. rufus fue la especie con mayor abundancia (22 registros) y en la época de lluvias $S$. floridanus la mejor representada con 32 registros (Figura 5). El índice de Shannon (H') fue mayor en la época de secas $\left(H^{\prime}=1.8 \pm 0.07\right)$ en contraste con la época de lluvias ( $\left.H^{\prime}=1.77 \pm 0.05\right)$, pero al comparar estos indicadores no se encontraron diferencias significativas $(t$-valor $=0.088$, g. I. $=190.85, P=0.92, n$. S.).

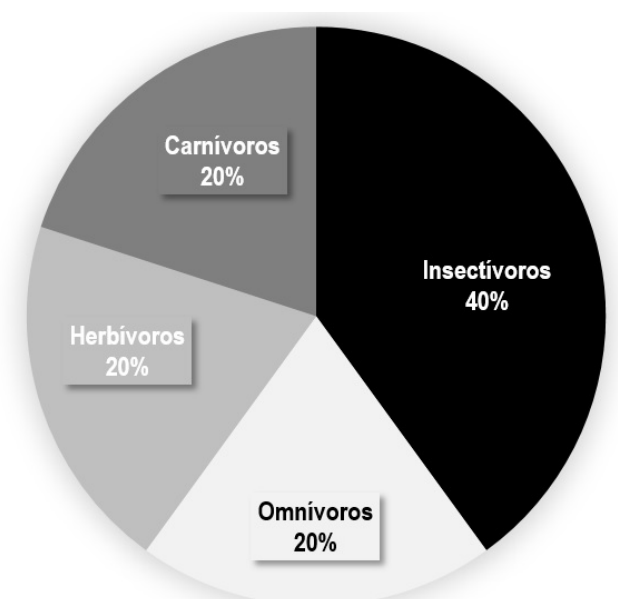

Figura 4. Gremios tróficos en los que se agrupan los mamíferos medianos registrados en el PNPO. 


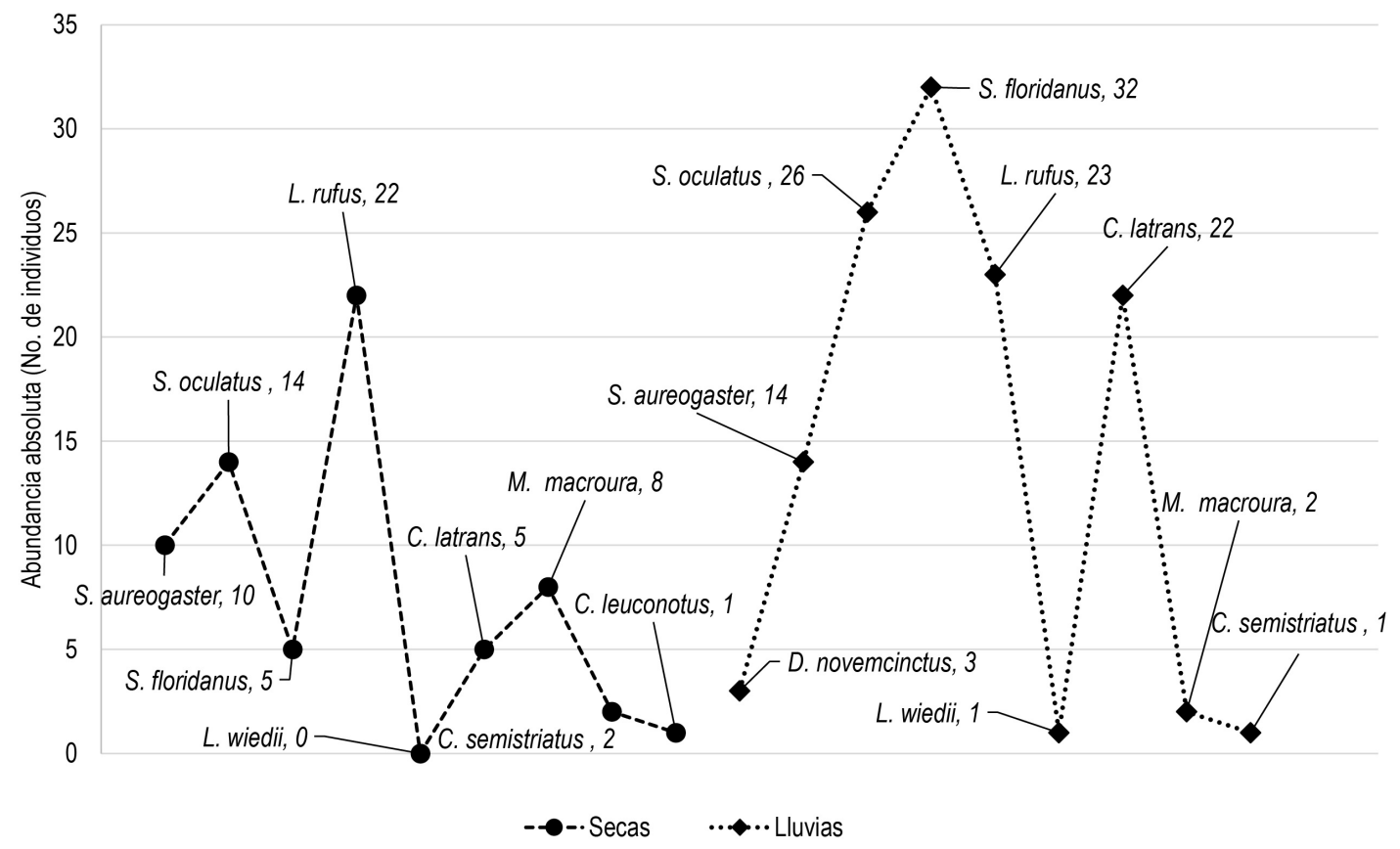

Figura 5. Curvas de rango-abundancia de la diversidad $\beta$ de mamíferos del PNPO registrados en la época de secas y lluvias. Los valores asociados a los nombres científicos representan su abundancia absoluta.

\section{DISCUSIÓN}

El Volcán Pico de Orizaba se encuentra al Este de la Faja Volcánica Transmexicana donde se reportan 15 especies de carnívoros, cinco de Lagomorfos, cuatro de Didelfimorfos y una del orden Cingulata (Gámez et al. 2012). En el presente estudio se registraron seis especies del orden Carnívora $(L$. rufus, L. weidii, C. latrans, M. macroura, C. semistriatus y $C$. leuconotus), un lagomorfo ( $S$. floridanus), dos del orden Rodentia (S. aureogaster y $S$. oculatus) y una del orden Cingulata ( $D$. novemcinctus), que también se han registrado al Este de la Faja Volcánica Transmexicana; el registro de estas especies en el PNPO representa el $40 \%$ de las especies reportadas por Gámez et al. (2012). A pesar de que en el PNPO se han realizado estudios sobre la diversidad de mamíferos desde hace más de tres décadas (Fa y Morales 1991) y se tiene conocimiento parcial de la diversidad de mamíferos que alberga esta ANP; este estudio representa el diagnóstico de una fracción de la diversidad de mamíferos que aún habita esta importante ANP, que en la actualidad se encuentra gravemente fragmentada y con impacto humano (Vázquez-Torres et al. 2010). Para complementar el inventario de riqueza mastofaunística que alberga el PNPO se requieren de estudios sobre diversidad de roedores y mamíferos voladores con la finalidad de tener un inventario más robusto, lo cual apoyaría a otras estrategias de conservación (González-Christen 2010). En el estado de Veracruz se reporta una riqueza de 195 de mamíferos entre los que destaca el orden Carnivora (22 especies), Rodentia (55 especies), Lagomorpha (5 especies) y una especie del orden Cingulata. A nivel estatal, las especies registradas en este estudio como los Carnívoros representan el 27\%, Rodentia el $3.6 \%$, Lagomorpha el $20 \%$ y Cingulata el $100 \%$ con respecto a los mamíferos reportados por GonzálezChristen y Delfín-Alfonso (2016) para el estado de Veracruz.

La completitud del inventario global de mamíferos medianos en este estudio fue superior al $95 \%$ en ambas épocas del año, lo que indica que el 
muestreo fue representativo en términos de la cobertura de la muestra. El grado de cobertura de la muestra de mamíferos medianos registrados en la época de secas y lluvias no fue estadísticamente diferente, es decir, la proporción que representan los individuos de cada especie con respecto al número total de individuos fueron similares entre las épocas del año (López-Mejía et al. 2017). Los resultados del presente estudio indican que la comunidad de mamíferos medianos en el PNPO no sufre cambios en términos de riqueza y abundancia entre épocas, lo que es indicador de una comunidad de mamíferos estable (Hernández-Hernández et al. 2018). Lo que es consistente con los resultados obtenidos con el indicador de Shannon $\left(\mathrm{H}^{\prime}\right)$, el cual no fue significativamente diferente con respecto a la riqueza y abundancia de mamíferos medianos registrados entre épocas. Esto puede deberse probablemente a que las especies registradas se encuentran en un hábitat favorable que les provee refugio, fuentes de agua, áreas de alimentación, zonas de reproducción y ruta de escape ante depredadores, por lo que el PNPO tiene un ecosistema que cubre las necesitas biológicas básicas de estas especies, a pesar de los disturbios humanos que ocurren en la zona, los cuales pueden alterar su comportamiento (Ávila-Nájera et al. 2018).

Al comparar la riqueza de mamíferos mediados registrados se encontró variación con la riqueza reportada en otras ANP y zonas con condiciones ecosistémicas templadas. Al respecto, en la Reserva Natural Sierra de Nachitla del estado de Morelos se reportan 19 especies de mamíferos medianos y grandes (Monroy-Vilchis et al. 2011). Por otra parte, en hábitats templados del sur de la Cuenca de México se han registrado 21 especies de mamíferos medianos (Monroy-Vilchis et al. 1999). En tanto que en el Parque Nacional Nevado de Toluca, México se registran 12 especies de mamíferos (SánchezJasso et al. 2013); mientras que en el Corredor Biológico Chichinautzin en Morelos se reportan 18 especies de mamíferos silvestres (Martínez et al. 2012). A pesar de que los estudios referidos superan la riqueza de mamíferos registrados para el PNPO, sobresale la ausencia de especies generalistas de hábitat como la zorra gris (Urocyon cinereoargenteus) y los tlacuaches (Didelphimorphia) que, como lo indica Rosenblatt et al. (1999), se puede interpretar como una medida indirecta de que el ecosistema que protege el PNPO aún conserva áreas con bajos impactos que alteren el hábitat de los mamíferos residentes (Ruiz-Soberanes y Gómez-Álvarez 2010, Rumiz 2010). La ausencia de grandes carnívoros se puede deber a que especies del gremio Carnívora requieren de grandes extensiones de hábitat y diversidad de presas disponibles para sobrevivir, por lo que la gestión del PNPO debe incrementar esfuerzos para aumentar la conectividad de hábitats que permita el recambio de estas especies y sus presas y con ello favorecer su permanencia (Serna-Lagunes et al. 2019).

Considerando que el grupo de los carnívoros fue el gremio más representado, con el $60 \%$ de mamíferos depredadores y $40 \%$ de presas, se infiere que el ecosistema no se encuentra en equilibrio trófico, ya que la proporción de los gremios en la red trófica no está balanceada (Silva 2005, MartínezVázquez et al. 2010). Los carnívoros con mayor abundancia (Figura 5 ) fueron $C$. latrans y $L$. rufus, los cuales posiblemente se alimenten de conejos, ardillas y posiblemente zorrillos y armadillos (Monroy et al. 2003, Cruz-Espinoza et al. 2008). Por lo que, para conservar las poblaciones de estos mamíferos, se deben implementar prácticas de mejoramiento del hábitat; por ejemplo, para conejos se pueden preservar y aumentar la superficie de zonas con presencia de pastizales, y para ardillas, conservar e incrementar las zonas donde se determine la menor incidencia de incendios forestales; esto con el fin de incrementar las poblaciones de las presas como alimento para depredadores. Con relación a la presencia de especies del orden Carnívora, Pérez-Irineo y SantosMoreno (2013) sugieren que su presencia se asocia con la diversidad y disponibilidad de alimento, y por lo tanto este es un orden complejo que presenta una alta diversidad de gremios tróficos. Aunado a esto, se ha documentado que la presencia y abundancia de depredadores afecta la dieta de carroñeros y herbívoros (Wilmers et al. 2003, McShea 2005), y que, sin los primeros, se puede llegar a una sobrepoblación de algunas especies, principalmente 
especies plagas y la disminución de otras, generando un desequilibrio en la estructura y función del ecosistema (Gittleman y Gompper 2005).

\section{CONCLUSIONES}

Se registraron 10 especies de ocho géneros que pertenecen a seis familias. El orden Carnívora fue el mejor representado con el $60 \%$ de las especies de mamíferos, por lo que se infiere que la red trófica no se encuentra balanceada. Tres especies de mamíferos se encuentran en riesgo: $L$. wiedii, C. semistriatus y $S$. oculatus, por tanto que los esfuerzos de conservación del PNPO deben orientarse al mejoramiento del hábitat de estas especies bajo el concepto de especies sombrilla. Se recomienda continuar con el monitoreo en el PNPO para tener un panorama claro de la riqueza y diversidad de mamíferos en esta área protegida.

\section{AGRADECIMIENTOS}

A la Unidad de Manejo y Conservación de Recursos Genéticos y al Laboratorio de Bioinformática y Bioestadística de la Facultad de Ciencias Biológicas y Agropecuarias región Orizaba-Córdoba, Universidad Veracruzana, que a través del proyecto Caracterización de recursos zoogenéticos de las altas montañas, Veracruz: aplicación de la filogeografía y modelación ecológica (PRODEP: 511-6/18-9245/PTC896), brindaron el apoyo técnico para el estudio. Al Editor asociado y los revisores anónimos que con sus observaciones ayudaron a mejorar el trabajo.

\section{LITERATURA CITADA}

Aranda MJ (2012) Manual para el rastreo de mamíferos terrestres de México. Comisión Nacional para el Conocimiento y Uso de la Biodiversidad. México. 255p.

Ávila-Nájera DM, Chávez C, Lazcano-Barrero MA, Mendoza GA, Pérez-Elizalde S (2016) Traslape en patrones de actividad entre grandes felinos y sus principales presas en el norte de Quintana Roo, México. Therya 7: 439-448.

Ávila-Nájera DM, Chávez C, Pérez-Elizalde S, Guzmán-Plazola RA, Mendoza GD, Lazcano-Barrero MA (2018) Ecology of Puma concolor (Carnivora: Felidae) in a Mexican tropical forest: adaptation to environmental disturbances. Revista de Biología Tropical 66: 78-90.

Ceballos G (2007) Conservation priorities for mammals in megadiverse Mexico: the efficiency of reserve networks. Ecological Applications 17: 569-578.

Ceballos G, Navarro D (1991) Diversity and conservation of Mexican mammals. In: Mares MA, Schmidly DJ (eds). Topics in Latin American mammalogy: History. Biodiversity, and Education. University of Oklahoma Press. Norman, Oklahoma. pp: 167-198.

Ceballos G, Oliva G (2005) Los mamíferos silvestres de México. Fondo de Cultura Económica. México. 986p.

Ceballos G, Rodríguez P, Medellín RA (1998) Assessing conservation priorities in megadiverse Mexico: mammalian diversity, endemicity, and endangerment. Ecological Applications 8: 8-17.

Cerano-Paredes J, Villanueva-Díaz J, Vázquez-Selem L, Cervantes-Martínez R, Esquivel-Arriaga G, Guerra-de la Cruz V (2016) Régimen histórico de incendios y su relación con el clima en un bosque de Pinus hartwegii al norte del estado de Puebla, México. Bosque 37: 389-399.

Chao A, Jost L (2012) Coverage-based rarefaction and extrapolation: standardizing samples by completeness rather than size. Ecology 93: 2533-2547. 
Chao A, Ma KH, Hsieh TC (2016) iNEXT (iNterpolation and EXTrapolation) online: Software for Interpolation and Extrapolation of Species Diversity. Program and User's Guide. http://chao.stat.nthu.edu.tw/wordpress/software_download/. Fecha de consulta: 13 de abril de 2019.

Chávez C, De La Torre A, Bárcenas H, Medellín RA, Zarza H, Ceballos G (2013) Manual de fototrampeo para estudio de fauna silvestre. El jaguar en México como estudio de caso. Alianza WWF-Telcel, Universidad Nacional Autónoma de México, México. 103p.

CONANP (2015) Programa de Manejo Parque Nacional Pico de Orizaba. Secretaria de Medio Ambiente y Recursos Naturales. México. Comisión Nacional de Areas Naturales Protegidas. México. 187p.

Cruz-Espinoza A, Pérez GEG, Santos-Moreno A (2008) Dieta del Coyote (Canis Latrans) en Ixtepeji, Sierra Madre de Oaxaca, México. Naturaleza y Desarrollo 8: 33-45.

Cumming G, Fidler F, Vaux DL (2007) Error bars in experimental biology. The Journal of Cell Biology 177: 7-11.

Danoff-Burg JA, Chen X (2005) Biodiversity calculator, abundance curve calculator. Columbia University. Disponible en: www.columbia.edu/itc/cerc/danoff-burg/Biodiversity\%20Calculator.xls. Fecha de consulta: 28 de mayo de 2018.

DOF (1937) Decreto Presidencial el día 4 de mayo de 1937, por el que se declaró Parque Nacional "Cofre de Perote" o "Nauhcampatépetl", la porción de los terrenos comprendidos en la parte superior de la gran montaña conocida con igual nombre, en el Estado de Veracruz. Diario Oficial de la Federación. México. Disponible en: https://www.dof.gob.mx/nota_detalle.php?codigo=5379137\&fecha=21/01/2015. Fecha de consulta: 15 de abril de 2019.

Díaz-Pulido A, Payán GE (2012) Manual de foto-trampeo: una herramienta de investigación para la conservación de la biodiversidad en Colombia. Instituto de Investigaciones de Recursos Biológicos Alexander von Humboldt y Panthera Colombia. Colombia. 32p.

Fa JE, Morales LM (1991) Mammals and protected areas in the Trans-Mexican Neovolcanic Belt. In: Mares MA, Schmidly DJ (eds). Latin American Mammalogy: history, biodiversity, and conservation. University of Oklahoma Press. Oklahoma, E.U. pp: 199-226.

Gámez N, Escalante T, Rodríguez G, Linaje M, Morrone JJ (2012) Caracterización biogeográfica de la Faja Volcánica Transmexicana y análisis de los patrones de distribución de su mastofauna. Revista Mexicana de Biodiversidad 83: 258-272.

García-Marmolejo G, Escalante T, Morrone JJ (2008) Establecimiento de prioridades para la conservación de mamíferos terrestres neotropicales de México. Mastozoología Neotropical 15: 41-65.

Gittleman J, Gompper ME (2005) The importance of carnivores for understanding patterns of biodiversity and extinction risk. In: Barbosa P, Castellanos I (eds.) Ecology of predators-prey interactions. Oxford University Press, Nueva York, EE.UU. pp: 330-388.

Goepel K (2012) True diversity-18.12.12.xIsx. California. USA. Disponible en: https://bpmsg.com/bpmsg-diversitycalculator-excel/. Fecha de consulta: 12 de febrero de 2018.

González-Christen A (2010) Los mamíferos de Veracruz. Guía ilustrada. Colección la Ciencia en Veracruz, Consejo Veracruzano de Investigación Científica y Desarrollo Tecnológico, Veracruz, México. 191p.

González-Christen A, Delfín-Alfonso CA (2016) Los mamíferos terrestres de Veracruz, México y su protección. En: Briones-Salas M, Hortelano-Moncada Y, Magaña-Cota G, Sánchez-Rojas G, Sosa-Escalante JE (eds.). 
Riqueza y conservación de los mamíferos en México a nivel estatal. Instituto de Biología, Universidad Nacional Autónoma de México, Asociación Mexicana de Mastozoología, Universidad de Guanajuato. Ciudad de México, México. pp: 499-534.

Gotelli NJ, Colwell RK (2001) Quantifying biodiversity: procedures and pitfalls in the measurement and comparison of species richness. Ecology Letters 4: 379-391.

Harrison RL (1997) Chemical attractants for Central American felids. Wildlife Society Bulletin 25: 93-97.

Hernández-Hernández JC, Chávez C, List R (2018) Diversidad y patrones de actividad de mamíferos medianos y grandes en la Reserva de la Biosfera La Encrucijada, Chiapas, México. Revista de Biología Tropical 66: 634-646.

Hill M (1973) Diversity and evenness: a unifying notation and its consequences. Ecology 54: 427-432.

LGEEPA (2007) Ley General del Equilibrio Ecológico y Protección al Ambiente. Diario Oficial de la Federación. México. 135p.

López-Mejía M, Moreno CE, Zuria I, Sánchez-Rojas G, Rojas-Martínez A (2017) Comparación de dos métodos para analizar la proporción de riqueza de especies entre comunidades: un ejemplo con murciélagos de selvas y hábitats modificados. Revista Mexicana de Biodiversidad 88: 183-191.

Magurran AE (1988) Ecological diversity and its measurement. Princeton University Press. New Jersey, EUA. $179 p$.

Magurran AE (2008) Measuring biological diversity. Wiley-Blackwell, Oxford, United Kingdom. 264p.

Martínez RM, Moreno JMP, García MAL, Flores AG (2012) Mastozoological study of the Chichinautzin biological corridor (COBIO), Morelos, Mexico. SITIENTIBUS série Ciências Biológicas 11: 16-23.

Martínez-Vázquez J, González-Monroy RM, Díaz-Díaz D (2010) Hábitos alimentarios del Coyote en el parque Nacional Pico de Orizaba. Therya 1: 145-154.

McSheA WJ (2005) Forest ecosystems without carnivores: when ungulates rule the world. In: Ray JC, Redford $\mathrm{KH}$, Steneck RS, Berger J (eds.). Large carnivores and the conservation of biodiversity. Island Press. Washington, EE.UU. pp: 138-153.

Mejía-Ronzón W, Mora-Brito AH, Villagómez-Cortés JA (2016) Turismo rural: una alternativa de agronegocios en la zona norte del Parque Nacional Pico de Orizaba. Ciencia Administrativa 5: 110-122.

Monroy VO, Ortega M, Velázquez A (2003) Dieta y abundancia relativa del coyote: un dispersor potencial de semillas. In: Velázquez A, Torres A, Bocco G (eds.). Las enseñanzas de San Juan. SEMARNAT-INE, Gobierno de Michoacán. Distrito Federal, México. pp: 565-591.

Monroy-Vilchis O, Rangel-Cordero H, Aranda M, Velázquez A, Romero FJ (1999) Los mamíferos de hábitat templados del sur de la Cuenca de México. En: Velázquez AA, Romero $F$ (eds.). Biodiversidad de la región de montaña del sur de la Cuenca de México. Universidad Autónoma Metropolitana, Secretaría de Medio Ambiente. México. pp: 141-159.

Monroy-Vilchis O, Zarco-González MM, Ramírez-Pulido J, Aguilera-Reyes U (2011) Diversidad de mamíferos de la Reserva Natural Sierra Nanchititla, México. Revista Mexicana de Biodiversidad 82: 237-248.

Morin PJ (2011) Community Ecology. Wiley-Blackwell. Nueva Jersey, EE. UU. 418p.

Pacheco LF, Guerra JF, Ríos-Uzeda B (2003) Eficiencia de atrayentes para carnívoros en bosques yungueños y praderas altoandinas en Bolivia. Mastozoología Neotropical 10: 167-176. 
Pérez-Irineo G, Santos-Moreno A (2013) Riqueza de especies y gremios tróficos de mamíferos carnívoros en una selva alta del sureste de México. Therya 4: 551-564.

Peterson AT, Egbert SL, Sánchez-Cordero V, Price KP (2000) Geographic analysis of conservation priority: endemic birds and mammals in Veracruz, Mexico. Biological Conservation 93: 85-94.

Ramírez-Pulido J, González-Ruiz N, Gardner AL, Arroyo-Cabrales J (2014) List of recent land mammals of Mexico, 2014. Natural Science Research Laboratory. Museum of Texas Tech University. USA. 69p.

Rosenblatt DL, Heske EJ, Nelson SL, Barber DM, Miller MA, MacAllister B (1999) Forest fragments in east-central Illinois: islands or habitat patches for mammals? The American Midland Naturalist 141: 115-123.

Ruiz-Soberanes JA, Gómez-Álvarez G (2010) Estudio mastofaunístico del Parque Nacional Malinche, Tlaxcala, México. Therya 1: 97-110.

Rumiz DI (2010) Roles ecológicos de los mamíferos medianos y grandes. In: Wallace RB, Gómez H, Porcel ZR, Rumiz DI (eds). Distribución, ecología y conservación de los mamíferos medianos y grandes de Bolivia. Centro de Ecología Difusión, Fundación Simón I. Patiño, Santa Cruz, Bolivia. pp: 53-73

Sánchez-Jasso JM, Aguilar-Miguel X, Medina-Castro JP, Sierra-Domínguez G (2013) Riqueza específica de vertebrados en un bosque reforestado del Parque Nacional Nevado de Toluca, México. Revista Mexicana de Biodiversidad 84: 360-373.

SARH (1993) Diagnóstico del Parque Nacional Pico de Orizaba, Veracruz. Secretaría de Agricultura y Recursos Hidráulicos. Subsecretaría Forestal y de Fauna Silvestre. Consultoría Multidisciplinaria. Veracruz. 86p.

SEMARNAT (2010) Norma Oficial Mexicana NOM-059-SEMARNAT-2010, Protección Ambiental-Especies nativas de México de Flora y Fauna Silvestres-Categorías de Riesgo y Especificaciones para su inclusión, exclusión o cambio-Lista de especies en riesgo. Diario Oficial de la Federación \#56, $2^{\mathrm{a}}$ Sección. México. $85 \mathrm{p}$.

Serna-Lagunes R, Álvarez-Oseguera LR, Ávila-Nájera DM, Leyva-Ovalle OR, Andrés-Meza P, Tigar B (2019) Temporal overlap in the activity of Lynx rugus and Canis latrans and their potential prey in the Pico de Orizaba National Park, Mexico. Animal Biodiversity and Conservation 42: 153-161.

Silva SI (2005) Posiciones tróficas de pequeños mamíferos en Chile: una revisión. Revista Chilena de Historia Natural 78: 589-599.

Simberloff D, Dayan T (1991) The guild concept and the ecological communities. Annual Reviews Ecology and Systematics 22: 115-143.

Vargas MF (1984) Parques Nacionales de México y Reservas equivalentes: pasado, presente y futuro. Colección: grandes problemas nacionales. Serie: Los Bosques de México, Instituto de Investigaciones Económicas. Universidad Nacional Autónoma de México. México. 266p.

Vázquez LB, Valenzuela-Galván D (2009) ¿Qué tan bien representados están los mamíferos mexicanos en la red federal de áreas naturales protegidas del país? Revista Mexicana de Biodiversidad 80: 249-258.

Vázquez-Torres SM, Carvajal-Hernández Cl, Aquino-Zapata AM (2010) Áreas naturales protegidas. In: Benítez BG, Welsh RC (eds.) Atlas del patrimonio natural, histórico y cultural de Veracruz. Patrimonio natural. Comisión del Estado de Veracruz para la Conmemoración de la Independencia Nacional y de la Revolución Mexicana, Gobierno del Estado de Veracruz, Universidad Veracruzana. Veracruz, México. pp: 249-274.

Wilmers CC, Crabtree RI, Smith DW, Murphy KM, Getz WM (2003) Trophic facilitation by introduced top predators: grey wolf subsidies to scavengers in Yellowstone National Park. Journal of Animal Ecology 72: 909-916. 
Wilson DE, Reeder DM (2005) Mammals species of the world; a taxonomic and geographic reference. Johns Hopkins University Press. Baltimore, EU. 2142p.

Wilson DE, Reeder DM (2011) Class Mammalia Linnaeus, 1758. Animal biodiversity: An outline of higher-level classification and survey of taxonomic richness. Zootaxa 3148: 56-60. 